\title{
Circumstellar dust shells around long-period variables
}

\section{Dynamics of C-rich AGB star shells dominated by the exterior $\kappa$-mechanism}

\author{
C. Dreyer, M. Hegmann, and E. Sedlmayr
}

\begin{abstract}
Technische Universität Berlin, Zentrum für Astronomie und Astrophysik (ZAA), EW 8-1, Hardenbergstr. 36, 10623 Berlin, Germany e-mail: dreyer@astro.physik.tu-berlin.de
\end{abstract}

Received 30 December 2008 / Accepted 25 February 2009

\section{ABSTRACT}

\begin{abstract}
Context. Miras and long-period variables (LPVs) are radially pulsating, highly evolved stars on the Asymptotic Giant Branch. Because of peculiar conditions of this objects, their cool, extended atmospheres are ideal sites for the formation of dust particles. Carbon-rich circumstellar dust shells (CDSs) surrounding stars with high stellar luminosity, tend to become dynamically unstable. They develop a self-maintaining oscillatory pattern caused entirely by dust formation even without the additional input of mechanical momentum from the star (exterior $\kappa$-mechanism). Since this system obviously has an eigenmode, it is interesting to consider the interaction with an exterior mechanical force, i.e. the radial pulsation at the inner boundary.

Aims. We investigate in great detail the complex dynamical behaviour of carbon-rich CDSs in a more systematic way. This is done by established methods of nonlinear dynamics.

Methods. We consider CDSs as multioscillatory systems that can be analysed with tools of nonlinear dynamics. We also use a discrete Fourier transform to examine the eigenmodes and their behaviour and apply this to a typical model CDS as a representative example. Results. In the absence of external excitation, the dynamics of the shell are dominated by its eigenmode, which is determined by the characteristic timescale of the coupled system of dust formation, hydrodynamics, and thermodynamics. The input mechanical energy and momentum of an underlying stellar pulsation introduces a new timescale to the system. Depending on the ratio of these two timescales, the dynamical behaviour of the system is either dominated by the eigenmode of the shell, by the excitation force, or can be irregular. In the latter case, the strength of the excitation becomes especially important. However, even for a small excitation period when the system is dominated by the shell's eigenmode, the oscillation of the shell tends to synchronise with the excitation force.
\end{abstract}

Key words. chaos - hydrodynamics - methods: numerical - stars: AGB and post-AGB - stars: oscillations stars: circumstellar matter

\section{Introduction}

Miras and long-period variables (LPVs) are highly evolved stars located on the asymptotic giant branch (AGB) with stellar luminosities as high as a few $10^{4} L_{\odot}$ and typical effective temperatures lower than $3000 \mathrm{~K}$, which show slow, massive stellar winds with final outflow velocities $u_{\infty}$ in the range of some $10 \mathrm{~km} \mathrm{~s}^{-1}$ and mass loss rates $\dot{M}$ as high as $10^{-4} M_{\odot} \mathrm{yr}^{-1}$. Modulated absorption of radiation in the stellar interior (internal $\kappa$-mechanism) causes large radial pulsations with periods in the range of approximately (100-1000) d (Whitelock et al. 1991), with a maximum by number at $350 \mathrm{~d}$ (Hoffmeister et al. 1990).

There has been a long-lasting debate about whether these stars pulsate in the fundamental mode or the first overtone (cf. Wood 1995). By comparing MACHO observations of LMC red giants with theoretical models, Wood et al. (1999) were able to provide strong evidence that LPVs on the MIRAS sequence are fundamental mode pulsators. However, the problem becomes even more intriguing, if the complex behaviour of the circumstellar dust shell (CDS) is also taken into account. Since the early work of Eddington \& Plakidis (1929), it has been known that the pulsations of Miras are not strictly regular, but show random cycle-to-cycle variations in period and, to a smaller degree, in amplitude. Percy \& Colivas (1999) analysed a database of times and magnitudes of maximum and minimum brightness of 391 bright Mira stars over a timeframe of 75 years, finding that the majority of the observed Mira light curves exhibit irregularities over intervals of about 20 cycles. Percy \& Bagby (1999) speculated that the observed variations on a timescale of 10-15 times the pulsation period are at least partially due to episodic dust emission.

In the context of the theory of dynamical systems, oscillating circumstellar shells may be conceived as multioscillatory systems, whose eigenfrequencies and normal modes are controlled by the intrinsic timescales of the various coupled physical and chemical processes. Because of the inherently nonlinear nature of the underlying equations, an element of randomness is added to the stellar pulsation (cf. Icke et al. 1992).

Confirmed by Höfner et al. (1996), Fleischer et al. (1995) demonstrated that an instability caused by the formation of dust (exterior $\kappa$-effect) can lead to self-induced oscillations of the CDS. This instability is controlled by the strong non-local feedback of newly formed dust that affects the temperature and dynamics of the circumstellar matter because of radiative transfer effects. As the first dust grains start to form in a specific temperature and density regime of the circumstellar shell, their subsequent rapid growth strongly increases the opacity in the dust-forming region. This process affects the dusty shell in two ways. On the one hand, radiative momentum is transferred effectively to the dust component and the gas is consequently accelerated in a mass-loss wind via frictional coupling. On the other hand, the recently formed grains absorb the stellar 
radiation very effectively. Isotropically re-emitted mainly in the infrared, it produces a pronounced heating of the material inside the dust layer. The higher temperature due to this backwarming not only prevents further nucleation of dust grains inside the dust shell, but also leads to a slight increase in the pressure of the CDS and a subsequent generation of small-amplitude waves in the region above the stellar atmosphere. Because of the density gradient in the shell, these subsonic disturbances steepen into shock fronts, which propagate outward leading to substantial mass-loss. Thereby, the dust density is diluted, producing a far lower opacity. Consequently, the stellar radiation is not absorbed effectively any longer. The resulting decrease in temperature enables a new cycle of dust formation and growth. This exterior $\kappa$-mechanism is a periodical phenomenon and its timescale $\tau_{\kappa}$ is the characteristic or eigenperiod $P_{K}$ of the atmosphere, which is inherent to the dust-forming system. It depends of course on the stellar parameters such as the abundance ratio of carbon to oxygen or the stellar luminosity. Extremely high luminosities and low temperatures are needed to sustain the oscillations of the CDS driven purely by the exterior $\kappa$-mechanism.

The interplay between the stellar radial pulsation and the exterior $\kappa$-mechanism leads to the complex dynamical behaviour of the CDS, which is the subject of our research. The aim of this article is to examine the characteristics of the CDS eigenmode and its interaction with the stellar pulsation. We investigate the complex dynamics of carbon-rich CDSs in a more systematic way than e.g., Fleischer et al. (1995), using a numerical algorithm of Discrete Fourier Transform (DFT) to determine the eigenmodes. Our analysis approach is completed by established tools of nonlinear dynamics, i.e., stroboscopic maps. We demonstrate the analysis method for a CDS model whose parameters were chosen to ensure that a stable periodic oscillation of the shell develops even without external excitation.

The paper is organised as follows. We start with a brief outline of our CDS modelling method in Sect. 2. The methods used to investigate the dynamical behaviour of CDSs are described in Sect. 3, and are applied to a representive model of a CDS in Sect. 4. We examine the characteristics of the CDSs eigenmode and its interaction with the stellar pulsation. This paper finishes with some concluding remarks and a short outlook in Sect. 5.

\section{Modelling of the CDS}

The approach used in this study to modelling the circumstellar dust-forming shells of C-rich Miras and LPVs is described in detail in Fleischer et al. (1992). The code includes the explicit solution of time-dependent hydrodynamics, thermodynamics, radiation transfer, equilibrium chemistry, and a detailed treatment of dust nucleation, growth, and evaporation (Gail \& Sedlmayr 1988) in Lagrangian coordinates. Starting from a given hydrostatic and dust-free initial situation, the purpose of the code is to yield a self-consistent model of both the time evolution and the spatial stratification of all relevant physical and chemical quantities determining the dynamical dust shell.

We consider a spherically symmetric atmosphere surrounding a pulsating red giant, characterised by the stellar mass $M_{*}$, the stellar temperature $T_{*}$, the stellar luminosity $L_{*}$, and the ratio of the photospheric abundance of carbon $\epsilon_{\mathrm{C}}$ to oxygen $\epsilon_{\mathrm{O}}$. To simulate the interior pulsation of the star, the so-called piston approximation (Wood 1979; Bowen 1988; Bowen \& Willson $1991)$ is applied. The velocity $u_{i}$ at the inner boundary $R_{i}$ oscillates sinusoidally in time with period $P$ and amplitude $\Delta u$. Consequently, the position of the inner boundary is described by a cosine variation about an equilibrium position $R_{0}$. Therefore, the models are defined by the prescription of the four fundamental stellar parameters $\left\{M_{*}, L_{*}, T_{*}, \epsilon_{C}\right\}$ and, in addition, by the two pulsation parameters $\{P, \Delta u\}$.

\section{Analysis approach}

A lucid and profound physical understanding of the system dynamics is provided by Fourier methods (e.g. Brigham 1974) by detecting the harmonics contained in a given signal. Since computational studies are never able to analyse continuous functions $h(t)$ but instead a list of measurements $h_{k}$ sampled at intervals in time $\Delta t$ (sampling interval), we used the Discrete Fourier Transform (DFT) to transform the signal from time space $(t)$ into frequency space $(f)^{1}$. The Fourier spectra of the amplitude $H\left(f_{n}\right)$ and of the power $\left|H\left(f_{n}\right)\right|^{2}$ are calculated according to:

$$
H\left(f_{n}\right)=\sum_{k=0}^{N-1} h_{k} \mathrm{e}^{2 \pi i k n / N} \Delta t \text { with } f_{n} \equiv \frac{n}{N \Delta t} \quad n=0, \ldots, N-1
$$

where $H\left(f_{n}\right)$ is the discrete Fourier transform of the $N$ points $h_{k}$.

\subsection{Sampling}

To avoid an aliasing problem, it is important that the signal is sampled with a frequency $f_{\mathrm{s}}=1 / \Delta t$ so that no information is close to the sampling frequency $f_{\mathrm{s}}$. According to the Sampling Theorem (see e.g., Brigham 1974), the highest frequency component of a sampled signal that can be correctly identified without aliasing is the "Nyquist critical frequency", given by

$f_{c}=\frac{1}{2 \Delta t}=\frac{f_{\mathrm{s}}}{2}$.

Any further increase in the signal frequency above the Nyquist value will result in an overlapping of spectra and contamination of data in the desirable region of the spectrum to be analysed. Aliasing may be minimised by increasing the sampling frequency $f_{\mathrm{s}}$. The DFT procedure gives the amplitudes of the sine and cosine components of the signal over the frequency baseband from 0 to $f_{c}$. We note, that this frequency baseband is fixed by the sampling process and not by the frequencies present in the signal.

\subsection{Spectral resolution}

The level of detail or resolution in the frequency spectrum is indicated by the spectral resolution $\Delta f$ :

$\Delta f=\frac{1}{N \Delta t}$.

To resolve small detailed structures in the spectrum of a signal, one needs to ensure that $N \Delta t$ is large, i.e., to sample the signal over a longer time. This can be achieved by either taking a large number of samples $N$, or by making the sampling interval $\Delta t$ long, or perhaps both. If the sampling interval is too long, however, aliasing of higher-frequency components of the signal may occur. On the other hand, increasing the number of samples has the disadvantage of increasing the computing time to obtain the DFT, and the amount of memory required to store the data during the processing. So one has to reach a reasonable compromise in choosing $\Delta t$ between computational time and the required accuracy of the analysis.

\footnotetext{
1 We note, that we prefer to write the transform in terms of oscillation frequency $f$ instead of angular frequency $\omega=2 \pi f$.
} 


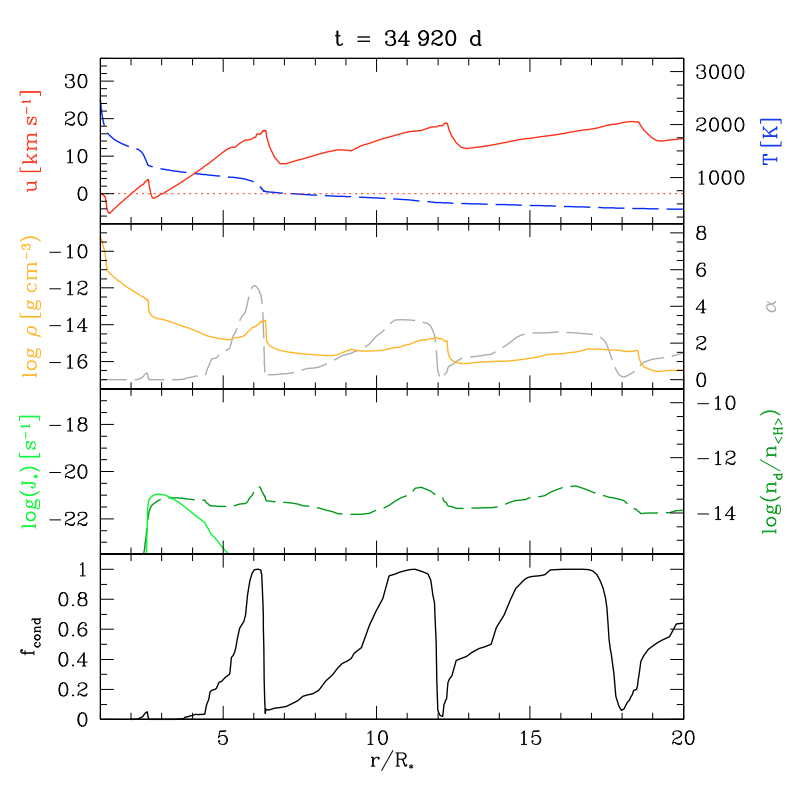

Fig. 1. Self-induced radial structure of our exemplary CDS: $L=3 \times$ $10^{4} L_{\odot} ; M=1 M_{\odot} ; T_{*}=2450 \mathrm{~K} ; \mathrm{C} / \mathrm{O}=1.25$. Upper panel: gas velocity $u$ (solid line), gas temperature $T$ (dashed line), second panel: mass density $\rho$ (solid line), radiative acceleration on dust in units of the local gravitational deceleration $\alpha$ (dashed line), third panel: stationary nucleation rate $J_{*}$ (solid line), the number of dust grains per $\mathrm{H}$-atom $n_{d} / n_{\langle\mathrm{H}\rangle}$ (dashed line), lower panel: degree of condensation $f_{\text {cond }}$ (solid line).

\section{Application}

\subsection{The CDS as oscillatory system}

Only for sufficiently high stellar luminosities $L_{*}$ can an oscillation of a CDS be sustained by the exterior $\kappa$-mechanism without the input of energy and momentum by an underlying pulsating stellar atmosphere.

To demonstrate and discuss the space-time evolution of the physical quantities of a CDS inferred by the numerical solution of the system of fundamental model equations, we plot the radial structure of the shell in an Eulerian presentation at one arbitrarily chosen instant of time to display a complete overall picture of the circumstellar dust shell. Figure 1 shows a snapshot of the radial structure of the fully developed circumstellar dust shell caused solely by the exterior $\kappa$-mechanism of an exemplary model with the parameters $L=3 \times 10^{4} L_{\odot}, M=1 M_{\odot}, T_{*}=2450 \mathrm{~K}$, and $\mathrm{C} / \mathrm{O}=1.25$. The dust-induced layered shell-structure can be seen for some characteristic hydrodynamical quantities, and quantities describing the dust complex.

The density gradient of the initial, hydrostatic structure of our high-luminosity model is gradual enough, i.e., the density scale height is sufficiently high to allow dust formation in the initial phase. A subsequent effective growth phase and radiation pressure acting on the newly formed dust grains both lead to the development of an outflow even without the additional input of mechanical energy. This is the onset of the instability solely caused by dust formation (exterior $\kappa$-mechanism). Due to the backwarming effect of the dust layer, the temperature below the shock front at $r \approx 2.5$ times the stellar radius $R_{*}$ increases. Consequently, this leads to a slight increase in pressure in the innermost region, and the subsequent generation of smallamplitude waves. The subsonic disturbances steepen because of the density gradient in the innermost region. The newly formed shock front travels outward and finally compresses the grains

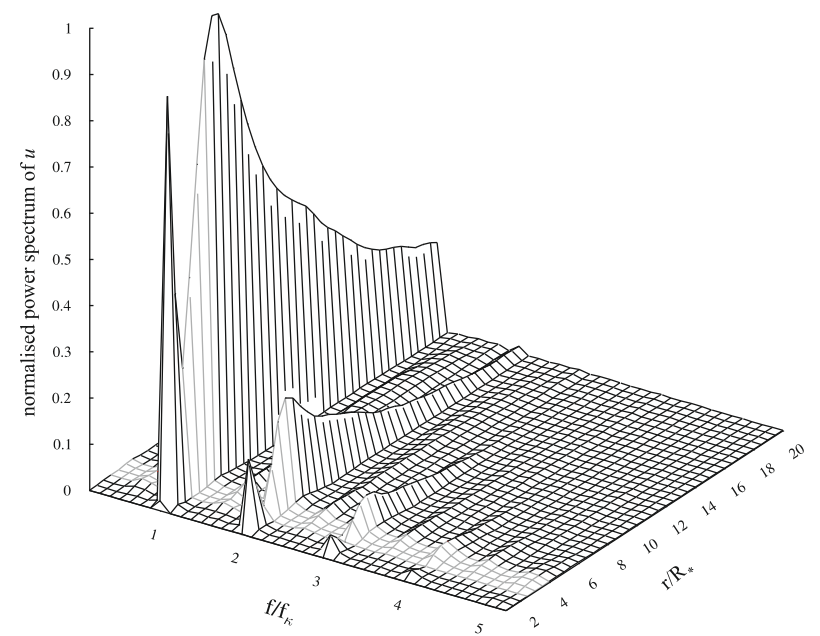

Fig. 2. Normalised power spectrum of the radial gas velocity $u$ with some well defined frequencies purely caused by exterior $\kappa$-mechanism. The position of the dust formation zone in the envelope is high lighted.

contained in the dust-forming region, thereby triggering the dust growth. The entire process starts again and results in a periodic outflow as seen in Fig. 1.

This representative CDS model was analysed by Fourier transforms, using the Cooley-Tukey algorithm (Press et al. 1986). This algorithm imposes the constraint that the number of samples must be equal to a power of $2\left(N=2^{\gamma}\right)$, where $\gamma$ is a positive integer. The velocity structure $u(t, r)$ of a circumstellar outflow is usually taken to be the characteristic tracer of (multi)periodicity. Consequently, our study is mainly focused on the detailed analysis of the velocity structure of the CDS model. With $\Delta t$ equidistantly sampled, the time-dependent velocity $u(t, r)$ was Fourier transformed at each fixed radial Eulerian coordinate $r$ for $1 R_{*} \leq r \leq 20 R_{*}$, where $R_{*}$ is the stellar radius. We chose a spatial resolution of $\Delta r=0.5 R_{*}$.

For $n=0$, the frequency corresponds to the zero-frequency component, caused by the upward shift of the signal (offset). The value of the offset has no effect on the spectrum, and the zero frequency component is therefore negligible.

Our approach considers only the most dominant frequencies, which were tested by grid studies using non-adaptive and adaptive grids with different numbers of gridpoints $j_{\max }=$ $\{1025,1500,1750,2000,2049,2500,3000,3072,3500,4097\}$ and grid rezoning or remapping rezoinc $=\{0.1 \mathrm{P}, 0.2 \mathrm{P}, 0.25 \mathrm{P}$, $0.3 \mathrm{P}, 0.314 \mathrm{P}, 0.5 \mathrm{P}, 0.7 \mathrm{P}, 1 \mathrm{P}\}$ (for details of rezoning see Fleischer et al. 1992) to ensure that no numerical influences dominate the physical structure of the CDS. To sample the time interval of about $t_{\max }=160000 \mathrm{~d}$, we used different sampling numbers $N=\{128,256,512,1024\}$, and sampling rates $\Delta t=\{38 \mathrm{~d}, 83 \mathrm{~d}, 97 \mathrm{~d}, 122 \mathrm{~d}, 155 \mathrm{~d}, 194 \mathrm{~d}, 272 \mathrm{~d}, 322 \mathrm{~d}$, $400 \mathrm{~d}$, to determine the optimal resolution. Some parameter combinations led to artificial oscillation modes (pseudofrequencies) because of the interaction of the redistributed grid with the sampling rate (cf. Sandin 2008). The optimal parameter combination for our model CDS was obtained for $\left\{j_{\max }=1025\right.$, rezoinc $=0.5 \mathrm{P}, N=512, \Delta t=83 \mathrm{~d}\}$, which corresponds to a Nyquist frequency of $f_{c}=0.006 \mathrm{~d}^{-1}$.

Figure 2 depicts the frequency behaviour of the selfsustaining oscillation for our representative CDS. We note that there is no internal pulsation of the star. The normalised power spectrum of the radial gas velocity $u$ is shown as a function of the radial coordinate $r$. The eigenfrequency $f_{\kappa}=(2459 \mathrm{~d})^{-1}$ and 
three of its overtones characterised by a monotonic decrease in strength with increasing frequency can be clearly identified. The amplitude of the eigenmode shows a strong dependence on the distance from the star. The minima at $r=2.5 R_{*}$ clearly evident for each eigenmode are particularly remarkable. A comparison with the radial trajectory in Fig. 1 shows that the position of these frequency-minima coincides with the dust nucleation zone located at $(2-3) R_{*}$. This represents the confirmation that the dust is responsible for creating these characteristic frequencies of the dust shell. Shortly below and beyond the rather narrow nucleation zone (marked light grey) these frequencies peak, according to the backward-directed dilution- and the forward-directed compression-wave.

The eigenperiod $P_{K}$ is determined by the characteristic timescale of the coupled system of dust formation, hydrodynamics, and thermodynamics within the dust-forming zone. The corresponding eigenfrequency $f_{\kappa}$ is simply given by $f_{\kappa}=1 / P_{\kappa}$.

\subsection{Periodically excited CDS}

The identification of an eigenmode of the dust-forming circumstellar shell raises the question of how its related oscillatory behaviour interacts with an additional excitation caused by the internal $\kappa$-mechanism. For this purpose, the response of the above defined model was studied for a series of sinusoidal variations with different periods $P$ and velocity amplitudes $\Delta u$ at the inner boundary describing the stellar pulsation.

Since the dynamics of CDSs are basically controlled by the dust, we focused in particular on the dust nucleation zone, which is usually located around $r \approx(2-4) R_{*}$. According to our representative model structure (cf. Fig. 1), it is located at $r=2.5 R_{*}$.

\subsubsection{Excitation period}

A linear system driven by an external periodic force will attempt to follow the applied force and hence oscillates with the applied frequency after a short, transient behaviour. This situation differs significantly from that of a nonlinear system like our CDS. Depending on the strength of its nonlinearity, the system shows a periodic, quasi-periodic, or chaotic behaviour.

The dependence of the oscillation period $P_{\mathrm{CDS}}$ of the shell in the dust nucleation zone on the excitation period $P$ is depicted in Fig. 3, where the ratio of the shell's oscillation frequency to the eigenfrequency $f_{\mathrm{CDS}} / f_{\kappa}$ is displayed for different excitation periods of constant velocity amplitude $\Delta u=1 \mathrm{~km} \mathrm{~s}^{-1}$. Three different regimes can be identified. For an excitation period of below approximately $P=1500 \mathrm{~d}$, the dynamics of the system is determined by the exterior $\kappa$-effect. The fundamental mode and its overtones can be clearly seen. As $P$ increases, they are shifted towards lower frequencies, become weaker in strength, and eventually vanish when their frequencies reach the excitation frequency $f=1 / P$. For $P>2300 \mathrm{~d}$, the CDS is dominated by the movement of the inner boundary. In-between those two regimes (shaded region), the behaviour of the system becomes irregular. However, some dominant frequencies can be identified. The piston appears to become more influential with increasing $P$. For $P=1500 \mathrm{~d}$, the piston frequency coincidences with the first harmonic of the eigenfrequency. Furthermore, it is remarkable that the fundamental mode exhibits a bifurcation at $P=1750 \mathrm{~d}$. Both branches are clearly distinguishable until the entire system is enslaved by the moving boundary for $P>2300 \mathrm{~d}$.

When the pulsation is switched on at the inner boundary, the input of mechanical energy and momentum introduces a new

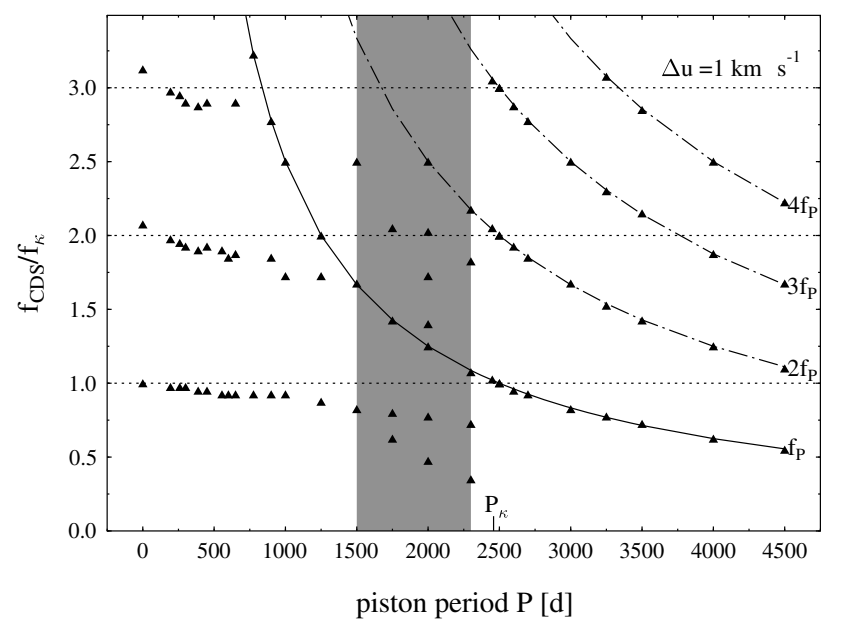

Fig. 3. Most dominant frequencies of a CDS excited with different piston periods $P$ near the dust nucleation zone $r=2.5 R_{*}$. The eigenperiod is labelled with $P_{K}$ and the irregular regime is marked in grey. The excitation frequencies (solid line) and their harmonics (dash-dotted lines), relative to $f_{\kappa}$, are also shown.

timescale to the system. In combination with the shocks already generated by the exterior $\kappa$-effect, the pulsation causes a further compression of the material to initiate dust growth. The radiation pressure acts on the newly formed dust and by means of the close momentum coupling between dust and gas, the twocomponent medium is accelerated outward. With increasing excitation period $P$, the additional input of energy and momentum becomes more significant. Close to the resonance period $P_{K}$, one observes a maximum transfer of energy, i.e., maximum amplitude. This leads to an enhanced density in the dust forming region. Consequently, the stellar wind becomes far more massive and the mass-loss rate changes from $\dot{M}=9.4 \times 10^{-5} M_{\odot} \mathrm{yr}^{-1}$ for the undisturbed CDS, to $\dot{M}=3.8 \times 10^{-4} M_{\odot} \mathrm{yr}^{-1}$ for the resonance situation. In contrast, the final outflow velocity remains almost constant at $u_{\infty} \approx 17 \mathrm{~km} \mathrm{~s}^{-1}$ compared to $u_{\infty} \approx 15.6 \mathrm{~km} \mathrm{~s}^{-1}$. As the wind becomes more intense, the periodic depletion in dust-forming material also increases and it takes more time to enrich the nucleation zone again. As a consequence, the eigenperiod becomes longer until the system finally becomes enslaved by the movement of the piston.

We now extend our analysis of the CDS by using established tools of nonlinear dynamics. For nonlinear systems, it is convenient to create so-called stroboscopic maps showing the time trajectory at discrete time intervals $\Delta t$. For a fixed position within the dust-forming zone, we projected the orbit in phase space onto the $(u, \rho)$ plane by choosing a time interval $\Delta t$ that is short relative to the excitation period $P$. For a system at a constant phase angle, i.e., after each period $P$, one obtains a special kind of stroboscopic map called a Poincare map. For more details of nonlinear dynamics, we refer the reader to, for example, Ott (2002).

Figure 4 shows power spectra and stroboscopic maps of the CDS for various periods $P=(1500 \mathrm{~d}, 900 \mathrm{~d}, 555 \mathrm{~d}, 388 \mathrm{~d})$ but with constant excitation-velocity amplitude $\Delta u=1 \mathrm{~km} \mathrm{~s}^{-1}$. For comparability, we normalised the different spectra to the same arbitrary unit. Cases of small amplitudes for short periods are depicted in greater detail in the inserted boxes of Figs. 4 and 6. The stroboscopic maps in the lower panels of the Fig. 4 indicate that the oscillation of the CDS synchronises with the excitation period to some extent. It can be seen that the system for a 

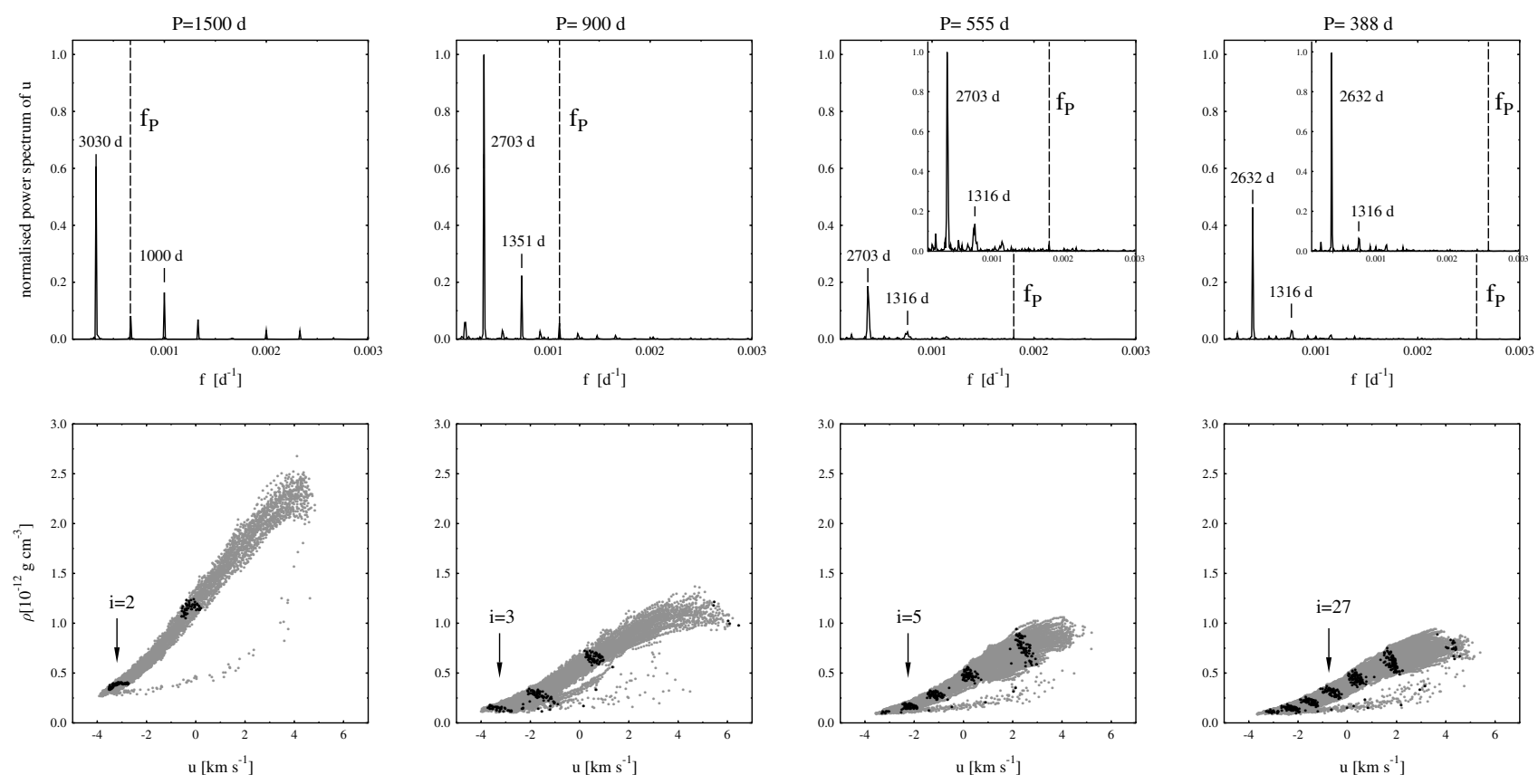

Fig. 4. Power spectra (upper panels) and stroboscopic maps (lower panels) for a CDS excited with different periods $P$ in the dust formig zone at $r=2.5 R_{*}$. The maps were obtained by plotting $(u(n 0.02 P), \rho(n 0.02 P))$ (grey) and $(u(n P), \rho(n P))$ (black) for $1 \leq n \leq\left\lfloor t_{\max } / P\right\rfloor, n \in \mathbb{N}$. Resonance stripes are marked with arrows.

constant phase angle (black dots) tends to stay inside a finite number of clearly demarcated stripes. In a similar way to the orbital resonance in celestial mechanics, two integers $i$ and $j$ can be found by fullfilling the condition $i P=j P_{\mathrm{CDS}}$. After $i$ periods $P$, the system approximately recaptures the same physical state, illustrated by one strip in the Poincare map (marked with arrow). The number of stripes $m$ increases with decreasing period $P$ and is roughly given by rounding $i / j=P_{\mathrm{CDS}} / P$ to the nearest integer.

For $P=388 \mathrm{~d}$ and $\Delta u=1 \mathrm{~km} \mathrm{~s}^{-1}$, for example, one finds that $i=27$. This means that the system passes through the same state every $27 \times 388 \mathrm{~d}$. Under the assumption $j=4$, this corresponds to $P_{\mathrm{CDS}}=2619 \mathrm{~d}$, which is confirmed to be $P_{\mathrm{CDS}}^{\mathrm{FFT}}=2632 \mathrm{~d}$ by a Fourier analysis of the temporal variation in the gas velocity $u$. The eigenmodes of the dust shell still determine the dynamics of the entire system. Due to the synchronisation, the response period $P_{\mathrm{CDS}}$ of the shell becomes only slightly longer than the period $P_{K}$ of the undisturbed system. Table 1 summarises the results for a series of excitation periods $P$ by giving the resonance integers $i, j, m$, and $P_{\mathrm{CDS}}$ and the most dominant periods $P_{\mathrm{CDS}}^{\mathrm{FFT}}$ determined by FFT. For some periods, no integers $i$ and $j$ could be found to satisfy the condition $i P=j P_{\mathrm{CDS}}$. Probably, $i P$ is similar or longer than the simulation time of the system for these excitation periods.

Figure 4 also underlines the statements mentioned at the beginning of this section concerning the mass-loss rates. With increasing piston period $P$, the velocity in the dust-nucleation zone is hardly effected, whereas the maximum density is strongly enhanced. Consequently, according to the equation of continuity $\dot{M}=4 \pi r^{2} \rho u$, the stellar wind becomes far more massive.

\subsubsection{Excitation strength}

To demonstrate the influence of the excitation strength, Fig. 5 presents the ratio $f_{\mathrm{CDS}} / f_{\kappa}$ and the corresponding overtones as a
Table 1. Resonance integers $i, j, m$ and resulting oscillation period $P_{\mathrm{CDS}}$ in comparison with the most dominant periods $P_{\mathrm{CDS}}^{\mathrm{FFT}}$ from the DFT of a CDS disturbed by different excitation periods $P$, but constant velocity amplitude $\Delta u=1 \mathrm{~km} \mathrm{~s}^{-1}$.

\begin{tabular}{rrrrrrrr}
\hline \hline$P[\mathrm{~d}]$ & $i$ & $j$ & $m$ & $P_{\mathrm{CDS}}[\mathrm{d}]$ & \multicolumn{3}{c}{$P_{\mathrm{CDS}}^{\mathrm{FFT}}[\mathrm{d}]$} \\
\hline 0 & - & - & - & - & 2459 & 1204 & 800 \\
194 & 13 & 1 & 13 & 2522 & 2564 & 1265 & 840 \\
259 & 10 & 1 & 10 & 2590 & 2564 & 1282 & 847 \\
300 & & & & & 2564 & 1299 & 862 \\
388 & 27 & 4 & 7 & 2619 & 2632 & 1316 & 870 \\
450 & 23 & 4 & 6 & 2588 & 2632 & 1299 & 862 \\
555 & 5 & 1 & 5 & 2775 & 2703 & 1316 & - \\
600 & 9 & 2 & 5 & 2700 & 2703 & 1351 & - \\
650 & & & & & 2703 & 1333 & 862 \\
776 & 7 & 2 & 4 & 2716 & 2703 & 776 & - \\
900 & 3 & 1 & 3 & 2700 & 2703 & 1351 & 901 \\
1000 & & & & & 2703 & 1449 & 1000 \\
1250 & & & & & 2857 & 1449 & 1250 \\
1500 & 2 & 1 & 2 & 3000 & 3030 & 1500 & 1000 \\
1750 & & & & & 4000 & 3125 & 1754 \\
2000 & & & & & 5263 & 3225 & 2000 \\
2300 & & & & & 7143 & 3448 & 2326 \\
\hline
\end{tabular}

function of the piston amplitude $\Delta u$ for $P=388 \mathrm{~d}$ (triangles), a representative period in which most of Miras pulsate, and for $P=1500$ d of the same order of magnitude as $P_{\mathrm{CDS}}$ (filled circles).

In the case of short pulsation periods, the oscillation frequencies $f_{\text {CDS }}$ decrease with increasing velocity amplitude and approach the value $f_{\mathrm{CDS}} / f_{\kappa} \approx 0.75$. Although a radial pulsation occurs at the inner boundary, the additional energy input of this external force has almost no influence on the timescale of the circumstellar shell. It barely has an impact on the mass-loss rate $\dot{M}$, which is enhanced from $9.4 \times 10^{-5} M_{\odot} \mathrm{yr}^{-1}$ (no excitation) to 


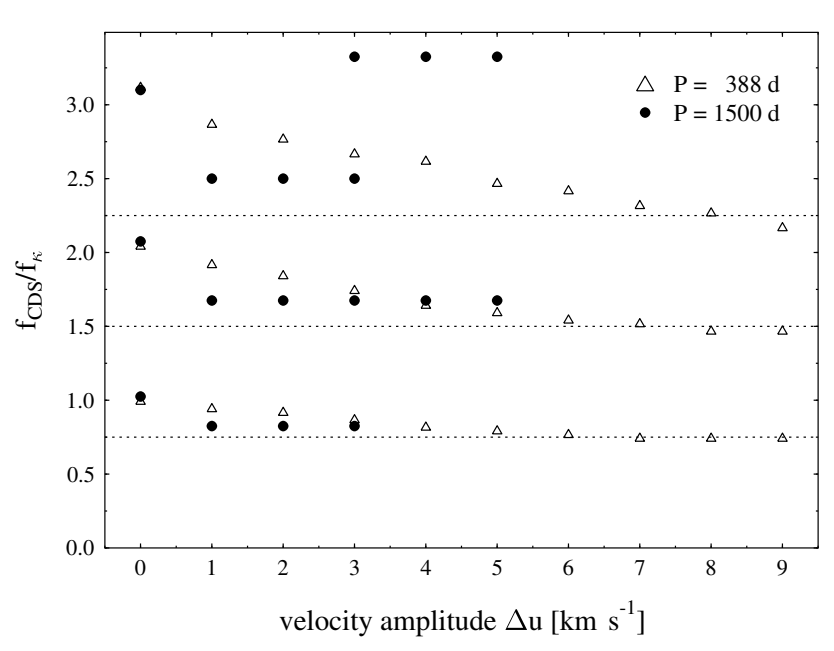

Fig. 5. Frequency shift of CDS eigenmodes $f_{\kappa}$ as a function of excitation strength $\Delta u$ using a fixed excitation period $P=388 \mathrm{~d}$ small compared to the eigenperiod (triangles), and for a larger excitation period $P=1500 \mathrm{~d}$ similar to the eigenperiod (filled circles) near nucleation zone.

$1.5 \times 10^{-4} M_{\odot} \mathrm{yr}^{-1}$ (resonance) at an approximately constant final outflow velocity of $u_{\infty} \approx 17 \mathrm{~km} \mathrm{~s}^{-1}$.

While the eigenmodes change only smoothly for a short period $P=388 \mathrm{~d}$, for a longer $P=1500 \mathrm{~d}$, the influence is a priori more significant. The frequencies are nearly synchronised, i.e., the eigenperiod is detuned to a lower harmonic of the excitation period and remains constant with increasing velocity amplitude. Finally, for a velocity amplitude $\Delta u \gtrsim 3 \mathrm{~km} \mathrm{~s}^{-1}$, the eigenmode completely vanishes and the excitation period and its harmonics dominate the system.

For a closer examination, we present in Fig. 6 spectra and stroboscopic maps for two different strengths of the excitation $\Delta u=1 \mathrm{~km} \mathrm{~s}^{-1}$ and $5 \mathrm{~km} \mathrm{~s}^{-1}$, using a fixed excitation period of $P=1500 \mathrm{~d}$. For the larger velocity amplitude $\Delta u=5 \mathrm{~km} \mathrm{~s}^{-1}$, the eigenmodes of the undisturbed CDS are replaced by the excitation period and its harmonics. The shell follows the exterior excitation identified by only one strip in the corresponding map, i.e. $i=j=1$. We note that the enhanced density results in a higher mass-loss rate $\dot{M}=7.9 \times 10^{-4} M_{\odot} \mathrm{yr}^{-1}$ and $u_{\infty} \approx 16.8 \mathrm{~km} \mathrm{~s}^{-1}$ compared to $\dot{M}=2.5 \times 10^{-4} M_{\odot} \mathrm{yr}^{-1}$ and $u_{\infty} \approx 15.6 \mathrm{~km} \mathrm{~s}^{-1}$ for $\Delta u=1 \mathrm{~km} \mathrm{~s}^{-1}$.

\section{Summary}

In the present paper, we have demonstrated a method for quantitatively analysing the dynamical behaviour of CDSs both in frequency and phase space and applied it to a model CDS with the stellar parameters $L=3 \times 10^{4} L_{\odot}, M=1 M_{\odot}, T_{*}=2450 \mathrm{~K}$, and $C / O=1.25$.

Even without any external perturbation by the internal $\kappa$ mechanism of the star, CDSs around high luminosity stars are able to accumulate a self-excited oscillation produced by the exterior $\kappa$-mechanism. The dynamics of the shell are dominated by its eigenmode, which is given by the characteristic dynamical timescales of the dust-formation zone. The eigenperiod of our model CDS was determined to be $P_{\kappa}=2459 \mathrm{~d}$ including a mass-loss of $\dot{M}=9.4 \times 10^{-5} M_{\odot} \mathrm{yr}^{-1}$ and a final outflow velocity of $u_{\infty} \approx 17 \mathrm{~km} \mathrm{~s}^{-1}$.
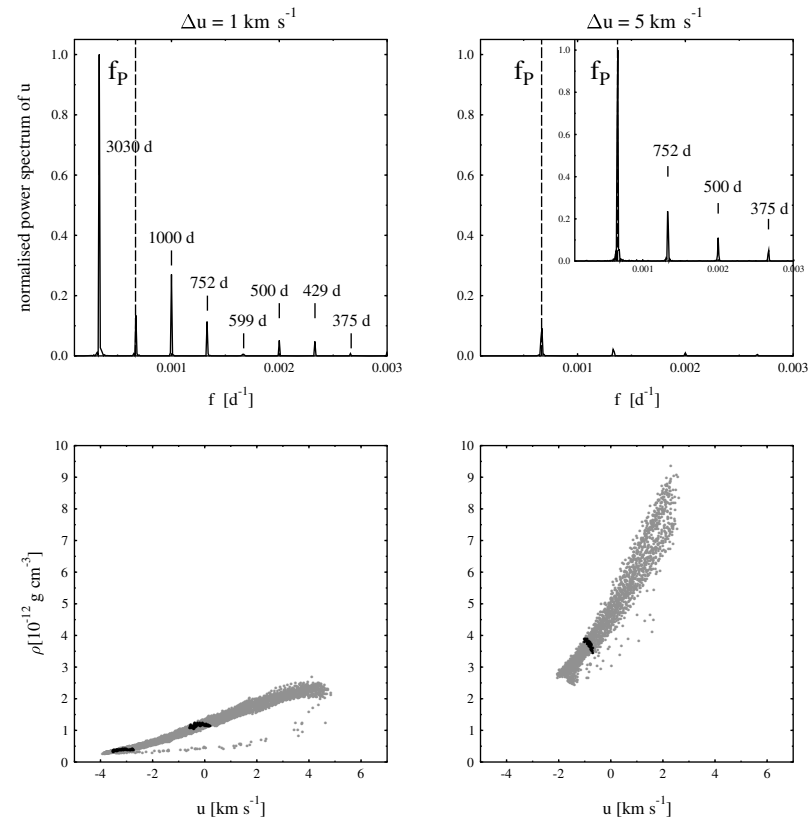

Fig. 6. Power spectra and stroboscopic maps for a CDS excited with a period $P=1500 \mathrm{~d}$ close to the eigenperiod, but with different strengths $\Delta u=1 \mathrm{~km} \mathrm{~s}^{-1}$ and $5 \mathrm{~km} \mathrm{~s}^{-1}$, respectively. The plot parameters for the maps are the same as in Fig. 4.

If excited by the internal $\kappa$-mechanism, the response of the shell depends strongly on the period $P$ of the excitation. For $P<P_{K}$, the dynamics of the shell remain dominated by its eigenmode. The eigenmode and its overtones are only shifted towards lower frequencies. With increasing excitation period, the piston gains influence, and the CDS reacts more irregularly. However, the Poincare maps show a pattern of discrete stripes, which indicate that the shell synchronises with the excitation period to some extent. For $P>P_{\kappa}$, the system becomes enslaved by the external excitation and the shell oscillates with period $P$.

For a small excitation period $P=388 \mathrm{~d}$, an increase in excitation strength results in a smooth shift towards lower frequencies. However, for an excitation period $P=1500 \mathrm{~d}$, an increase in excitation strength changes the dynamics of CDS from eigenperiod- to piston period-dominated.

The condition for a purely dust-driven wind by the exterior $\kappa$-mechanism is evident only at the very end of the AGB evolution. If the stellar luminosity decreases, the dominance of the dust is diminished. Further reduction eventually leads to a vanishing outflow, if no additional energy support, e.g., pulsation is provided. In the case of lower stellar luminosities, the system generates no self-induced shocks by the exterior $\kappa$-mechanism. Therefore, the eigenmodes of the system cannot be directly determined. The examination of the complex behaviour of these systems and a more systematic parameter study will be the subject of forthcoming publications.

Acknowledgements. Part of this work was supported by the Deutsche Forschungsgemeinschaft, DFG in the frame of SFB 555.

\section{References}

Brigham, E. O. 1974, The fast Fourier transform (Englewood Cliffs, NJ, Prentice-Hall)

Bowen, G. H. 1988, ApJ, 329, 299 
Bowen, G. H., \& Willson, L. A. 1991, ApJ, 375, L53

Eddington, A. S., \& Plakidis, L. 1929, MNRAS, 90, 65

Fleischer, A. J., Gauger, A., \& Sedlmayr, E. 1992, A\&A, 266, 321

Fleischer A. J., Gauger, A., \& Sedlmayr, E. 1995, A\&A, 297, 543

Gail, H. P., \& Sedlmayr, E. 1988, A\&A, 206, 153

Höfner, S., Feuchtinger, M. U., \& Dorfi, E. A. 1995, A\&A, 297, 815

Icke, V., Frank, A., \& Heske, A. 1992, A\&A, 258, 341

Hoffmeister, C., Richter, G., \& Wenzel, W. 1990, Veränderliche Sterne, 3rd ed.,

J. A. Barth (Leipzig)

Ott, E. 2002, Chaos in Dynamical systems, 2nd edn. (Cambridge University Press)
Percy, J. R., \& Colivas, T. 1999, PASP, 111, 94

Percy, J. R., \& Bagby, D. A. 1999, PASP, 111, 203

Press, W. H. Flannery, B. P., Teukolsky, S. A., \& Vetterling, W. T. 1986,

Numerical Recipes (Cambridge University Press)

Sandin, C. 2008, MNRAS, 385, 215

Whitelock, P. A., Feast, M. W., \& Catchpole, R. M. 1991, MNRAS, 248, 276

Wood, P. R. 1979, ApJ, 227, 220

Wood, P. R. 1995, ASP Conf. Ser., 83

Wood, P. R., Alcock, C., Allsman, R. A., et al. 1999, in Asymptotic Giant Branch Stars, ed. T. Le Bertre, A. Lebre, \& C. Waelkens, IAU Symp., 191, 151 